\title{
HIV Seroprevalence Among Patients Admitted to Two Psychiatric Hospitals
}

\author{
Francine Cournos, M.D., Maureen Empfield, M.D., Ewald Horwath, M.D., \\ Karen McKinnon, M.A., Ilan Meyer, M.A., M.Phil., Howell Schrage, M.D., \\ Cathie Currie, Ph.D., and Brenda Agosin, M.A.
}

\begin{abstract}
Objective: The authors determined the seroprevalence of HIV-1 among patients admitted to two psychiatric hospitals in New York City. Method: Patients consecutively admitted to an acute psychiatric unit in Manhattan and a large state hospital in Queens were anonymously tested for HIV-1 antibodies from December 1989 through July 1990. Test results were linked to age, gender, ethnicity, and two risk behaviors: male homosexual activity and injection drug use. Results: Blood was obtained from $83.0 \%$ of the eligible patients. The prevalence of HIV was $5.5 \%$ (25 of 451 ). Black patients accounted for $38.0 \%$ of the patients tested and $76.0 \%$ of positive results $(N=19)$, a rate of $11.1 \%$ for this group. The rate of seropositivity was comparable in women and men. Clinicians had charted risk behavior for nine (36.0\%) of the 25 HIV-positive patients. Infection control records suggested that clinicians were aware of seven (28.0\%) of the positive cases. Conclusions: One in every 18 patients admitted to two public psychiatric hospitals in New York City was HIV positive. Clinical staff largely failed to identify HIV-positive patients. Ethnicity and a history of homosexual activity among men or use of injected drugs were strongly associated with seropositivity. This pattern of infection may be linked to needle sharing and/or sexual activity with partners who have shared needles. Future research should clarify how psychiatric illness affects risk-taking behavior, focus on improving detection by clinicians, and identify effective prevention strategies in this population.

(Am J Psychiatry 1991; 148:1225-1230)
\end{abstract}

W e have seen an alarming increase in HIV infection among the severely mentally ill. The 4,000-bed state hospital system in New York City identified its first case of AIDS in 1983. By 1988, 24 patients who were openly tested that year were positive for HIV-1 antibodies, and in 1989 the number had increased to 53 newly tested HIV-positive patients (1). In addition to known cases, an unknown number of infected psychiatric patients with risk-taking histories who do not consent to HIV antibody testing or who do not report risk behaviors are being cared for in psychiatric hospitals.

Presented at the 143rd annual meeting of the American Psychiatric Association, New York, May 12-17, 1990. Received Nov. 6, 1990; revision received Feb. 19, 1991; accepted March 18, 1991. From the New York State Psychiatric Institute and Washington Heights Community Service, New York. Address reprint requests to Dr. Cournos, New York State Psychiatric Institute, 722 West 168th St., New York, NY 10032.

This study was funded by NIMH grant MH-46251 and the New York State Office of Mental Health.

The authors thank Diane Engel, M.S.W., Michael Georgescu, Diana Hartel, Ph.D., Nancy Hopkins, R.N., Jim Johnson, Ph.D., Ellie Schoenbaum, M.D., and Zena Stein, Ph.D.

Copyright 191991 American Psychiatric Association.
The severely mentally ill as a group are vulnerable to HIV infection because of comorbidity with substance abuse (2). Psychiatric diagnosis and substance abuse appear to be related (3-6), leading many investigators to hypothesize a self-medication effect $(3,5-7)$. Chronic substance abuse may also precipitate and exacerbate psychosis $(8,9)$. Smokable cocaine (crack) and injection drugs are among the substances used by psychiatric patients and are highly associated with HIV infection (10-15).

Unsafe sexual behavior, including sex with strangers or with partners who inject drugs, has also been reported among the severely mentally ill (16-23), and psychiatric symptoms are likely to affect patients' perceptions of their own HIV risk and efficacy in preventing infection (24).

At present little is known about the prevalence of HIV infection among psychiatric patients. Zamperetti et al. (25) reported that $6.5 \%$ of 475 patients admitted to a psychiatric ward in Milan over 2 years were HIV positive. In an ongoing study of patients admitted to acute care psychiatric units in a private hospital in New York City, Sacks et al. found that of 254 patients between the ages of 18 and 55 years who were anony- 
mously tested for HIV-1, 20 were positive, a rate of 7.9\% (VI International Conference on AIDS, 1990). One-half of the seropositive patients reported male homosexual activity, and one-quarter reported either injection drug use or sex with a partner who injected drugs. In another ongoing study, nine $(7.3 \%)$ of 124 patients admitted to a psychiatric unit for the homeless were reported positive for HIV-1 (Empfield et al., VI International Conference on AIDS, 1990).

\section{METHOD}

\section{Patients and Procedure}

We tracked patients between the ages of 18 and 59 years who were consecutively admitted to two psychiatric hospitals in New York City from December 1989 through July 1990 . One site was a 22-bed acute care unit at an academic medical center in northern Manhattan mandated to accept all patients referred from the local catchment area; 133 eligible patients were admitted to this unit. The other site was a large state hospital providing acute and chronic care to the borough of Queens; 413 eligible patients were admitted here. Both settings are state funded and mandated to treat severe mental illness. Most patients are either uninsured or have Medicaid. Any patient with a primary diagnosis of either dementia or substance abuse is excluded by policy from admission.

Following current federal regulations for anonymous sampling (26), we obtained discard blood samples from blood drawn for routine purposes at the time of admission. Patients whose discard blood was not obtained on admission were tracked for subsequent blood workup. Samples could not be obtained for every patient because of patient refusal of blood tests, patient discharge before blood drawing, or insufficient quantity. Blood samples were obtained for 456 of the 546 patients. We kept a record of every patient admitted so that we would obtain only a single sample of blood from each patient even if individual patients were admitted more than once during the study period. This patient roster was destroyed after all the discard blood samples were obtained.

Patient chart number, date of birth, admission diagnosis, and number of previous psychiatric hospitalizations were obtained from each patient's chart and recorded on the top half of a two-part form to obtain profiles of the eligible patients unlinked with HIV status. Age range, gender, ethnicity, known use of injected drugs, and history of homosexual activity among men were obtained from the patient chart and recorded on the lower half of the form. Because few Asian patients are admitted to either hospital, Asians were grouped with Caucasians to avoid the possibility of identifying any HIV-positive Asian patient. After demographic and risk factors were ascertained, the patient identification (upper) section of the form was removed and a six-digit bar-code number was affixed to the lower section to link the blood sample with the demographic and risk factor data.

The blood samples were stored, frozen, and sent in batches of at least 25 to the New York Blood Center for antibody testing. Specimens that were shown to be reactive by enzyme-linked immunosorbent assay (ELISA) were tested with the Western Blot and then classified according to current recommendations of the Centers for Disease Control (27). A specimen was considered positive if antibodies to two of the following were detected: p24, gp41, and gp120/160. The presence of any single band on the Western Blot constituted an indeterminate result. Because the sampling was anonymous, obtaining a second specimen for retesting from patients whose test results were inconclusive was not possible.

\section{Data Analysis}

A group profile of the tested patients, unlinked to their blood specimens, provided descriptive clinical data, including admission diagnosis and number of previous psychiatric hospitalizations. Linking of the blood test results with the patients' demographic characteristics and HIV risk behaviors provided prevalence rates within subgroups. All data were measured at either a nominal or ordinal level; therefore, contingency table analysis was used to interpret the data. Chi-square and Bartholomew's tests were performed, and odds ratios were used to estimate relative risk. Adjusted odds ratios were derived from logistic regression coefficients. Statistical significance was expressed in exact values, with the exception of the result of the Bartholomew's test, which was expressed as significant or not significant. The criterion for significance was set at an alpha level of 0.05 .

\section{RESULTS}

Of the 456 samples sent to the New York Blood Center for antibody testing, three were reported to be too small for testing. Of the 453 samples tested, $28(6.2 \%)$ were reactive according to ELISA. Two of these showed indeterminate results on Western Blot and were omitted from the analysis of relative risk and serostatus. Of the remaining 26 samples that were reactive on ELISA, 25 were confirmed positive by Western Blot. This results in a seroprevalence rate of $5.5 \%$ ( 25 of 451$)$. The distribution of positive results is shown in table 1 . One sample that was reactive according to ELISA was negative according to Western Blot.

Of the 133 eligible patients admitted to the 22-bed Manhattan unit during the study period, 128 were tested for HIV. Of the 413 eligible patients admitted to the state hospital in Queens during the study period, 325 were tested. Eight $(6.3 \%)$ of the 128 tested samples from the acute unit were positive, and $17(5.2 \%)$ of the 325 tested samples from the state hospital in Queens were positive. The rates at the two sites were not statis- 
TABLE 1. Association of HIV Infection With Injection Drug Use, Homosexual Activity Among Men, and Demographic Factors in 451 Psychiatric Patients

\begin{tabular}{|c|c|c|c|c|c|c|c|c|c|c|c|}
\hline \multirow{4}{*}{ Variable } & \multirow{4}{*}{$\begin{array}{c}\text { Patients } \\
\text { Tested }^{\mathrm{a}}\end{array}$} & \multirow{3}{*}{\multicolumn{2}{|c|}{$\begin{array}{l}\text { HIV-1 } \\
\text { Antibody } \\
\text { Positive }\end{array}$}} & \multirow{3}{*}{\multicolumn{3}{|c|}{$\begin{array}{c}\text { Chi-Square } \\
\text { Analysis }\end{array}$}} & \multicolumn{2}{|c|}{$\begin{array}{l}\text { Unadjusted } \\
\text { Odds Ratio } \\
\end{array}$} & \multicolumn{3}{|c|}{$\begin{array}{l}\text { Adjusted } \\
\text { Odds Ratio }\end{array}$} \\
\hline & & & & & & & \multirow{3}{*}{$\begin{array}{l}\text { Odds } \\
\text { Ratio }\end{array}$} & \multirow{3}{*}{$\begin{array}{c}95 \% \\
\text { Confidence } \\
\text { Interval }\end{array}$} & \multirow{3}{*}{$\begin{array}{l}\text { Odds } \\
\text { Ratio }\end{array}$} & \multirow{3}{*}{$\begin{array}{c}95 \% \\
\text { Confidence } \\
\text { Interval }\end{array}$} & \multirow[b]{3}{*}{$\mathbf{p}$} \\
\hline & & & & & & & & & & & \\
\hline & & $\mathrm{N}$ & $\%$ & $x^{2}$ & $\mathrm{df}$ & $\mathrm{p}$ & & & & & \\
\hline Age (years) ${ }^{b}$ & & & & $0.46^{c}$ & 3 & n.s. & 1.35 & $0.55-3.32$ & 1.27 & $0.48-3.34$ & 0.62 \\
\hline $18-29$ & 142 & 9 & 6.34 & & & & & & & & \\
\hline $30-39$ & 155 & 9 & 5.81 & & & & & & & & \\
\hline $40-49$ & 110 & 5 & 4.55 & & & & & & & & \\
\hline $50-59$ & 44 & 2 & 4.55 & & & & & & & & \\
\hline Ethnicity $^{\mathrm{d}}$ & & & & 17.79 & 2 & 0.0001 & 5.63 & $2.20-14.40$ & 6.33 & $2.39-16.73$ & 0.0002 \\
\hline Black & 172 & 19 & 11.05 & & & & & & & & \\
\hline Hispanic & 115 & 5 & 4.35 & & & & & & & & \\
\hline Caucasian/Asian & 163 & 1 & 0.61 & & & & & & & & \\
\hline Sex & & & & 0.22 & 1 & 0.64 & 1.21 & $0.54-2.72$ & 2.23 & $0.84-5.93$ & 0.11 \\
\hline Male & 237 & 12 & 5.06 & & & & & & & & \\
\hline Female & 214 & 13 & 6.07 & & & & & & & & \\
\hline Injection drug use & & & & $5.97^{e}$ & 1 & 0.02 & 3.76 & $1.41-10.06$ & - & - & - \\
\hline Identified & 39 & 6 & 15.38 & & & & & & & & \\
\hline Not identified & 412 & 19 & 4.61 & & & & & & & & \\
\hline $\begin{array}{l}\text { Homosexual activity } \\
\text { in men }(N=237)\end{array}$ & & & & $3.16^{e}$ & 1 & 0.08 & 4.67 & $1.14-19.07$ & - & - & - \\
\hline Identified & 18 & 3 & 16.67 & & & & & & & & \\
\hline Not identified & 219 & 9 & 4.11 & & & & & & & & \\
\hline Any risk factor & & & & $12.18^{e}$ & 1 & 0.0005 & 4.76 & $1.99-11.40$ & 7.58 & $2.69-21.36$ & 0.0001 \\
\hline Identified & 54 & 9 & 16.67 & & & & & & & & \\
\hline Not identified & 397 & 16 & 4.03 & & & & & & & & \\
\hline Total & 451 & 25 & 5.54 & & & & & & & & \\
\hline
\end{tabular}

${ }^{\mathrm{a}}$ Two patients had indeterminate results and were omitted from this analysis.

${ }^{b}$ Comparison groups for calculating the odds ratios for age were 18-39 and 40-49 years.

'Bartholomew's test for increasing proportions (trend).

$\mathrm{N}=450$ because ethnicity was not recorded for one seronegative patient. Comparison groups for calculating the odds ratios for ethnicity were black and nonblack.

${ }^{\mathrm{e}}$ With continuity correction.

tically different $\left(\chi^{2}=0.18, d f=1, p=0.67\right)$. Site as a variable was omitted from further analysis because of this nonsignificant difference.

In total, we tested 453 of the 546 admitted patients, or $83.0 \%$. There were no significant differences in age or gender between the patients whose blood we obtained and those we did not. Caucasian and Asian patients were represented somewhat less among the blood samples collected than among those not collected. Blood was not obtained for $15.5 \%$ of the black patients and $10.0 \%$ of the Hispanics, whereas for the Caucasian/ Asian group, blood samples for $21.6 \%$ were not obtained $\left(x^{2}=8.12, \mathrm{df}=2, \mathrm{p}=0.02\right)$.

As a group, the 456 sampled patients had the following admission diagnoses: schizophrenia, $\mathrm{N}=\mathbf{2 0 6}$ $(45.2 \%)$; schizoaffective disorder, $\mathrm{N}=39$ (8.6\%); affective disorder, $\mathrm{N}=114(25.0 \%)$; other psychotic disorders, $\mathrm{N}=53(11.6 \%)$; all other diagnoses, $\mathrm{N}=42(9.2 \%)$, including two patients with unknown diagnoses. Number of known previous psychiatric hospitalizations was as follows: zero hospitalizations, $\mathrm{N}=65(14.3 \%)$; one hospitalization, $\mathrm{N}=49(10.7 \%)$; two to five hospitalizations, $\mathrm{N}=297$ (65.1\%); more than five hospitalizations, $\mathrm{N}=45(9.9 \%)$. There were no significant differences be- tween the sampled patients and the patients whose blood was not obtained in diagnosis $\left(x^{2}=0.72, d f=3\right.$, $\mathrm{p}=0.87$ ) or number of previous hospitalizations $(\mathrm{t}=0.44, \mathrm{df}=109.8, \mathrm{p}=0.66)$.

When we examined the linked risk histories of the seropositive patients, clinicians had identified risk behavior in nine $(36.0 \%)$ of the 25 patients. Among the 12 HIV-positive men, six $(50.0 \%)$ had known risk behaviors. Three $(25.0 \%)$ used injected drugs, and three $(25.0 \%)$ had histories of homosexual activity. Among the 13 HIV-positive women, three $(23.1 \%)$ injected drugs. Of the 428 seronegative patients, 33 (7.7\%) were identified as injection drug users. Of the 225 seronegative men, $15(6.7 \%)$ had histories of homosexual activity. Relative risk estimations were derived with unadjusted odds ratios to compare the risks of testing positive for HIV-1 antibodies among the patients with and without identified risk factors. Unadjusted odds ratios calculate relative risk for each factor without controlling for effects of other risk variables. The unadjusted odds ratios showed that identified injection drug users were almost four times as likely to be seropositive as patients without histories of injection drug use. Overall, the risk of being seropositive was five times as 
high for the patients with known histories of at least one of the measured risk behaviors as for those who did not have such histories. The association of serostatus with demographic and behavioral risk factors is presented in table 1.

Using multiple logistic regression to simultaneously analyze the effects of several independent variables on HIV status, we found ethnic group and behavioral risk factors to independently contribute to the likelihood of being HIV seropositive. The adjusted odds ratios show that the risk for blacks was six times that of subjects in other ethnic groups, and the chance of having a positive Western Blot result for patients with one or more behavioral risks was seven and one-half times the risk for other patients.

The unadjusted and adjusted estimations of relative risk demonstrate essentially the same relationship between risk factors and HIV seropositivity. Thus, ethnicity and behavioral risk factors independently increase the likelihood of testing positive for HIV-1 antibodies.

\section{DISCUSSION}

Most of the patients in our study were psychotic and had multiple psychiatric admissions. The most common clinical diagnosis was schizophrenia, and $75.0 \%$ of the patients had had two or more hospitalizations before the index admission. The $5.5 \%$ prevalence of HIV indicates that certain severely mentally ill patients are at risk for HIV infection. A history of risk behavior was charted by clinicians for only $36.0 \%$ of the seropositive patients. Despite this low rate of recorded risk, a history of risk behavior, either homosexual activity among men or injection drug use, predicted HIV seropositivity.

The results of this study suggest several conclusions regarding the pattern of HIV infection among an urban, severely and chronically ill psychiatric population. First, the highest rates of infection are among black patients. In our group, one in nine black patients was HIV positive. Second, women are as likely to be infected as men. Third, a record of risk behavior is likely to be found for only a minority of patients.

Having learned that women in this population are as likely to be infected as men, we must make active efforts to identify their risk-taking behaviors, which include having sexual partners who inject drugs. Recent data (28) show that for New Jersey and New York, HIV/ AIDS is the leading cause of death for black women aged 15-44 years. This HIV/AIDS death rate is nine times that for Caucasian women of the same age. It is not clear to what extent differences in infection rates between black, Hispanic, and white women are caused by differences in risk behavior, exposure to infected sexual or needle-sharing partners, and biological vulnerability to HIV infection.

The pattern of infection we found also suggests a link to injection drug use. The New York City Department of Health (29) reported that for $49.7 \%$ of the black and Hispanic men in New York City with known cases of
AIDS, use of injected drugs is the primary risk factor. Among all women with AIDS in New York City, injection of drugs is the primary risk factor for $61.5 \%$ and sex with men at risk is the primary risk factor for $24.1 \%$. In contrast, among white men with AIDS, only $12.4 \%$ report injection of drugs as their primary risk behavior. Given that in our group $76.0 \%$ of the seropositive subjects were black and $52.0 \%$ were women, injection of drugs and having sexual partners who inject drugs are likely to be major factors in how psychiatric patients acquire HIV infection.

The rate of HIV infection in our subjects does not simply mirror other rates in the New York City area. The rate of $5.5 \%$ in our subjects is intermediate among reported rates in a number of clinical settings in New York City. For example, the New York City Department of Health (30) performed prevalence studies in 1988-1989 and found the following lower rates of HIV infection among 6,325 tested patients: abortion clinics, $1.6 \%$; sentinel hospital A, $1.4 \%$; sentinel hospital B, $2.7 \%$. A 1988 study of the mentally retarded living in the metropolitan New York City region (31) showed no HIV-positive subjects among 241 clients whose blood was tested. On the other hand, our rate was lower than that reported for the high-risk patients seen in New York City clinics for sexually transmitted diseases (32). There, $8.8 \%$ of 8,931 patients tested in 1988 and 1989 were positive for HIV-1 infection.

Our study sites are in areas of New York City with low to moderate rates of AIDS cases. In June 1990, the rates of AIDS cases in the city varied by zip code from 57 cases to 1,802 cases per 100,000 population (29). The range in the neighborhoods we drew our patients from was 65 to 348 cases per 100,000 population. Significantly higher rates might be found on psychiatric units with patients from areas of the city with higher rates of AIDS. The reported seroprevalence rate is, nevertheless, an estimate of the minimum percent of AIDS cases we will eventually see in our population.

Anonymous seroprevalence studies have advantages and disadvantages. An anonymous study does not identify the patient or require that any extra blood be drawn. Because there is no impact on the patient, consent is not required. This method allows investigators to test larger groups of patients, thereby increasing statistical power and representativeness without self-selection bias and without interfering with clinical judgment about the risks and benefits of testing for any individual patient. Patients who might otherwise refuse HIV testing or be unable to give informed consent can be included. On the other hand, because the study is not performed with the consent of the patient, it is impossible to conduct structured diagnostic or risk assessment interviews. Our study therefore had to depend on the information available in hospital records. We did not link blood samples to psychiatric diagnosis because admission diagnoses are not as reliable as those established by research interviews. We did not want to draw erroneous conclusions about the relationship between HIV status and psychiatric diagnosis. 
The anonymous survey method poses an ethical problem because it determines the presence of HIVpositive people within a group without allowing identification, counseling, and medical care. Identification of HIV-positive psychiatric patients might result in additional stigmatization of this chronically and severely ill population. These disadvantages had to be weighed against the value of establishing their prevalence of HIV. We concluded that the information gained would be essential to planning for the needs of a large number of severely mentally ill patients and therefore the benefits of obtaining a full epidemiological picture outweighed the disadvantages of the method.

Sampling limitations also may have affected our results. Because Caucasians and Asians were overrepresented among the $17.0 \%$ of admitted patients for whom we did not obtain blood, we may have overestimated HIV prevalence in our subjects. On the other hand, our findings probably underestimate the prevalence of HIV-1 among psychiatric patients from neighborhoods in New York City with higher concentrations of HIV. We did not extract chart information about sexual partners who injected drugs or were HIV positive. The existing literature led us to bias our questionnaire in favor of recording risk behavior among men. In addition, chart review may have underestimated the clinicians' knowledge of their patients. Clinical staff may have had knowledge about risk behavior that they did not record, either to maintain confidentiality or because it seemed irrelevant for charting purposes.

Independent of our study, the clinicians at the two hospitals involved in our investigation are required to report known HIV-positive patients to hospital infection control nurses. During the same period in which our anonymous study determined 25 patients to be HIV positive, the infection control nurses recorded seven HIV-positive patients. We cannot validate the accuracy of the infection control records, and we do not know whether any of the seven reported cases were among our patients for whom blood samples were not available. However, this observation suggests that, at best, clinicians were aware of only seven of the $25 \mathrm{HIV}$-positive patients identified by the anonymous survey.

Clinical staff at the large state hospital reported two HIV-positive patients to infection control committees. This compares to the 17 seropositive patients who were found by the anonymous study. On the small acute care unit, five HIV-positive patients were reported by clinical staff to the infection control committee, and the anonymous study found eight seropositive patients. Therefore, the low rates of risk behavior charted by clinicians seem consistent with the small number of HIVpositive patients identified by them.

Risk behaviors were detected for all the HIV-positive patients reported to the infection control committees. Of the seven patients who were known to be HIV positive, two $(28.6 \%)$ were men and five $(71.4 \%)$ were women. One man was identified as having injected drugs, and one had a female sexual partner with HIV/ AIDS. One of these patients was black, and one was
Hispanic. Of the five women identified by clinicians as HIV positive, two were black and three were Hispanic. Among the women, three were identified as users of injected drugs, three used cocaine, one had a history of prostitution, and four were identified as having sexual partners who injected drugs. It is clear that once someone tests positive for HIV, risk-taking behaviors are more aggressively investigated. However, many infected people are passing through undetected, making early intervention impossible.

When risk taking is identified, appropriate counseling and serologic testing with patient consent is indicated. This should be done in the context of clinical care. We believe the issue of mandatory testing is not one that the mental health system should attempt to address independently. Psychiatric patients deserve the same consideration as other groups when individual rights and public health interests are balanced. For most groups, the current approach is to encourage testing with informed consent but not to require it. Given sufficient clinical attention, many infected patients can be identified. The small acute care unit in our study, which has a high physician-to-patient ratio, was more successful at this task than the large state hospital, which has less physician oversight. However, even at the state hospital, it may be possible to overcome this problem by developing systematic procedures for inquiring about HIV-risk behavior.

In an ongoing study, we are collecting seroprevalence data from homeless and long-stay units and risk assessment interview data from multiple units at the two hospitals. These data will allow us to develop a prediction model for HIV infection and AIDS cases and to examine how psychiatric symptoms and risk are related. We hope to find ways to protect the considerable number of risk-taking psychiatric patients who are antibody negative and to reduce the risk of transmission by those who are already infected. We believe these efforts are uniquely within the province of psychiatry and urge additional efforts in this area.

\section{REFERENCES}

1. Central Office Infection Control Reports, 1988-1989. Albany, New York State Office of Mental Health, 1989

2. Way BB, McCormick LL: The Mentally Ill Chemical Abusing Population: A Review of the Literature. Albany, New York State Office of Mental Health, June 1990

3. Lehman AF, Myers CP, Corty E: Assessment and classification of patients with psychiatric and substance abuse syndromes. Hosp Community Psychiatry 1989; 40:1019-1025

4. Richard ML, Liskow BI, Perry PJ: Recent psychostimulant use in hospitalized schizophrenics. J Clin Psychiatry 1985; 46:79-83

5. Schneier FR, Siris SG: A review of psychoactive substance use and abuse in schizophrenia: patterns of drug choice. J Nerv Ment Dis 1987; 175:641-652

6. Khantzian EJ: The self-medication hypothesis of addictive disorders: focus on heroin and cocaine dependence. Am J Psychiatry 1985; 142:1259-1264

7. Kosten TR, Kleber HD: Differential diagnosis of psychiatric comorbidity in substance abusers. J Subst Abuse Treat 1988; 5 : 201-206

8. Safer DJ: Substance abuse by young adult chronic patients. Hosp Community Psychiatry 1987; 38:511-514 
9. Sheets JL, Prevost JA, Reihman J: Young adult chronic patients: three hypothesized subgroups. Hosp Community Psychiatry 1982; 33:197-203

10. Hasbrouck LM, Daniels N, Bowser BP, Gross SA: Crack-related behaviors undermining AIDS prevention among intravenous drug users, in Abstracts, VI International Conference on AIDS. Los Angeles, American Foundation for AIDS Research, 1990, p 271

11. Schoenbaum EE, Hartel D, Friedland GH: Crack use predicts incident HIV seroconversion. VI International Conference on AIDS, THC 1990; 103:159

12. McLellan AT, Druley KA: Random relation between drugs of abuse and psychiatric diagnoses. J Psychiatr Res 1977; 13:179184

13. Estroff TW, Dackis CA, Gold MS, Pottash AL: Drug abuse and bipolar disorders. Int J Psychiatry Med 1985-1986; 15:37-40

14. Atkinson R: Importance of alcohol and drug abuse in psychiatric emergencies. Calif Med 1973; 118:1-4

15. Alterman AI, Erdlen DL, Laporte DJ: Effects of illicit drug use in an inpatient psychiatric population. Addict Behav 1982; 7:231-242

16. Cournos F, Empfield M, Horwath E, Schrage H: HIV infection in state hospitals: case reports and long-term strategies. Hosp Community Psychiatry 1990; 41:163-166

17. Gewirtz GR, Horwath E, Cournos F, Empfield M: Patients at risk for HIV (letter). Hosp Community Psychiatry 1988; 39: 1311-1312

18. Horwath E, Kramer M, Cournos F, Empfield M, Gewirtz G: Clinical presentations of AIDS and HIV infection in state psychiatric facilities. Hosp Community Psychiatry 1989; 40:502-506

19. Sacks M, Dermatis H, Ott S, Perry S: HIV seroprevalence in psychiatric inpatients, in Abstracts, VI International Conference on AIDS. Los Angeles, American Foundation for AIDS Research, 1990, p 178

20. Zverina J, Lackman M, Pondelickova J, Vanek J: The occurrence of atypical sexual experience among various female patient groups. Arch Sex Behav 1987; 16:321-326

21. Akhtar S, Thomson JA: Schizophrenia and sexuality: a review and report of twelve unusual cases-part II. J Clin Psychiatry 1980; 41:166-174

22. Verhulst J, Schneidman B: Schizophrenia and sexual functioning. Hosp Community Psychiatry 1981; 32:259-262

23. Lukoff D, Gioia-Hasik D, Sullivan G, Golden JS, Nuechterlein $\mathrm{KH}$ : Sex education and rehabilitation with schizophrenic male outpatients. Schizophr Bull 1986; 12:669-677

24. Ostrow DG: AIDS prevention through effective education. Daedalus 1989; 118:229-254

25. Zamperetti M, Goldwurm GF, Abbate E, Gris T, Muratori S, Vigo B: Attempted suicide and HIV infection: epidemiological aspects in a psychiatric ward, in Abstracts, VI International Conference on AIDS. Los Angeles, American Foundation for AIDS Research, 1990, p 182

26. OPRR Reports: Regulation 45 CFR 46.102 (f). Washington, DC, US Department of Health and Human Services, Office of Protection from Research Risks, 1985

27. Association of State and Territorial Public Health Laboratory Directors and AIDS Program, Centers for Disease Control: Interpretation and use of the Western blot assay for serodiagnosis of human immunodeficiency virus type 1 infections. MMWR 1987; 36:509-515

28. Chu SY, Buehler JW, Berkelman RL: Impact of the human immunodeficiency virus epidemic on mortality in women of reproductive age, United States. JAMA 1990; 264:225-229

29. AIDS Surveillance Update, June 1990. New York, Department of Health AIDS Surveillance Unit, 1990

30. AIDS Surveillance Update, March 1990. New York, Department of Health AIDS Surveillance Unit, 1990

31. Pincus SH, Schoenbaum EE, Webber M: A seroprevalence survey for human immunodeficiency virus antibody in mentally retarded adults. NY State J Med 1990; 90:139-142

32. Weisfuse IB, Chiasson MA, Back S, Ramirez L, Greenberg B, Ewing W: HIV-1 infection in NYC STD clinic patients: evidence for stable seroprevalence, 1987-1988, in Abstracts, V International Conference on AIDS. Los Angeles, American Foundation for AIDS Research, 1989, p 89 